\title{
ON THE SOBCZYK-HAMMER DECOMPOSITION OF ADDITIVE SET FUNCTIONS
}

\author{
WILFRIED SIEBE
}

\begin{abstract}
It is observed that continuity for charges is equivalent to the absence of two-valued minorants. This characterization forms the basis of a new short proof within a functional-analytic context of a decomposition theorem by A. Sobczyk and P. C. Hammer [5] for charges on a field $\mathfrak{H}$ into a continuous part and a part which can be written as a sum of at most two-valued charges on $\mathfrak{H}$. A counterexample shows that in general the decomposition of a charge into a nonatomic part and a part which has no nonnull nonatomic minorant is not unique.
\end{abstract}

1. Definitions. Let $\mathfrak{A}$ be a field of subsets of a set $\Omega$. A charge $\mu$ on $\mathfrak{A}$ is a real-valued nonnegative finitely additive function defined on $\mathfrak{A}$. A measure is a countably additive charge whose domain is a $\sigma$-field of subsets of a set. A set $A \in \mathfrak{A}$ will be called an atom for $\mu$ iff $\mu(A)>0$ and for every set $E \in \mathfrak{A}, E \subset A$, either $\mu(E)=0$ or $\mu(E)=\mu(A) . \mu$ is nonatomic iff there are no atoms for $\mu$. Finally, a charge $\mu$ on a field $\mathfrak{U}$ (in $\Omega$ ) is said to be continuous iff, given $\varepsilon>0$, there exists a partition $P=\left\{B_{1}, \ldots, B_{n}\right\}$ of $\Omega$ into a finite number of pairwise disjoint members of $\mathfrak{A}$ such that $\mu\left(B_{i}\right)<\varepsilon$ for every $i$. Let $\mathfrak{B}$ denote the family of all such partitions, and $|P(\mu)|$ the maximum of $\mu\left(B_{i}\right)$ over all parts $B_{i}$ of $P \in \mathfrak{B}$.

2. Main result. It is well known that a measure $\lambda$ on a $\sigma$-field $\subseteq$ can be decomposed uniquely into $\lambda=\lambda_{0}+\lambda^{\prime}$ where $\lambda^{\prime}$ is a nonatomic measure on $\subseteq$ and $\lambda_{0}$ a completely atomic measure on $\mathfrak{S}$, i.e., $\lambda_{0}$ has the form $\lambda_{0}=\sum_{i=1}^{\infty} \lambda_{i}$ with at most two-valued measures $\lambda_{i}$ on $\subseteq(i \in \mathbf{N})$.

A. Sobczyk and P. C. Hammer have proved an analogous decomposition theorem for charges on fields of subsets of a set:

Decomposition Theorem (SobczyK-Hammer [5, Theorem 4.1]). Let $\mu$ be a charge on a field $\mathfrak{A}$ (in a set $\Omega$ ). Then $\mu$ can be decomposed uniquely into $\mu=\mu_{0}+\mu^{\prime}$ with a continuous charge $\mu^{\prime}$ on $\mathfrak{A}$ and a charge $\mu_{0}$ on $\mathfrak{A}$ which has the form $\mu_{0}=\sum_{i=1}^{\infty} \mu_{i}$ with at most two-valued charges $\mu_{i}$ on $\mathfrak{A}(i \in \mathbf{N})$.

The following lemma yields a reformulation of this theorem, for which a short functional-analytic proof will be given. The Krein-Milman representation theorem will serve as a link to the classical Lebesgue decomposition theorem.

Received by the editors April 15, 1981.

1980 Mathematics Subject Classification. Primary 28A10; Secondary 28A45.

Key words and phrases. Continuous resp. nonatomic charges, Krein-Milman representation theorem, decomposition theorem. 
Lemma. Let $\nu$ be a charge on a field $\mathfrak{A}$. $\nu$ is continuous iff $\nu$ admits no two-valued minorant, i.e., $\nu$ has the property $(*) \nu \geqslant \eta$ implies $\eta=0$ for any at most two-valued charge $\eta$ on $\mathfrak{A}$.

Proof. A canonical indirect argument shows that a continuous charge on $\mathfrak{A}$ also has the property $(*)$. Now suppose that $\nu$ is not continuous. Then by Lemmas 4.1 and 4.2 of [5] there exists a two-valued charge $\nu^{*}$ on $\mathfrak{A}$ with $\nu \geqslant \nu^{*}$ : Firstly, $\alpha=\inf _{P \in \mathfrak{B}}|P(\nu)|>0$ implies the existence of a set $\Omega_{0} \in \mathfrak{U}$ with (i) $\alpha \leqslant \nu\left(\Omega_{0}\right)<2 \alpha$, and such that every partition $P \in \mathfrak{P}$ contains some part $B_{i}$ with (ii) $\alpha \leqslant \nu\left(B_{i} \Omega_{0}\right)$. Notice that for any $\varepsilon>0$ there is a partition $P_{0} \in \mathfrak{B}$ with $\left|P_{0}(\nu)\right|<\alpha+\varepsilon$. Choose $\varepsilon<\alpha$. Then by $\alpha=\inf _{P \in \mathfrak{B}}|P(\nu)|>0$ the family $\left\{C_{1}, \ldots, C_{k}\right\}$ of members from $P_{0}$ with $\alpha \leqslant \nu\left(C_{i}\right)<2 \alpha$ for every $i$ is nonempty and has a member $\Omega_{0}$ such that for every partition $P=\left\{B_{1}, \ldots, B_{n}\right\} \in \mathfrak{B}$ there is an index $i$ with $\alpha \leqslant \nu\left(B_{i} \Omega_{0}\right)$. Secondly, let $\nu^{*}$ be defined on $\mathfrak{A}$ by $\nu^{*}(A)=\alpha$, resp. 0 , if $\nu\left(A \Omega_{0}\right) \geqslant \alpha$, resp. $\nu\left(A \Omega_{0}\right)<\alpha$, for every $A \in \mathfrak{A}$. Then $\nu^{*}$ is the desired two-valued charge: Consider disjoint sets $A$, $B \in \mathfrak{A}$ with $\nu\left((A+B) \Omega_{0}\right) \geqslant \alpha$. Then property (i) implies $\nu\left(\left(\Omega_{0} \backslash A \Omega_{0}\right) \backslash B \Omega_{0}\right)<\alpha$. Thus by (ii) either $\nu\left(A \Omega_{0}\right) \geqslant \alpha$ or $\nu\left(B \Omega_{0}\right) \geqslant \alpha$ (but not both, by (i) again). Hence $\nu^{*}(A+B)=\alpha=\nu^{*}(A)+\nu^{*}(B)$, which finishes the proof.

TheOREM. Let $\mu$ be a charge on a field $\mathfrak{A}$ of subsets of a set $\Omega$. Then $\mu$ is decomposable uniquely into $\mu=\mu_{0}+\mu^{\prime}$ where $\mu^{\prime}$ is a charge on $\mathfrak{A}$ with property (*) and $\mu_{0}$ is a charge on $\mathfrak{A}$ of the form $\mu_{0}=\sum_{i=1}^{\infty} \mu_{i}$ with at most two-valued charges $\mu_{i}$ on $\mathfrak{U}(i \in \mathbf{N})$.

Proof. Existence. Let $\mu$ be a probability charge on $\mathfrak{A}$. Let $X$ denote the set of all probability charges on $\mathfrak{A}$ and $X_{e}$ the set of extreme points of $X$. Then by the theorem of Krein-Milman [3, p. 6] there exists a regular probability measure $\rho$ on the Borel sets of $X$ with $\rho\left(X_{e}\right)=1$ and the property $\mu(A)=\int_{X_{e}} \nu(A) \rho(d \nu)$ for every $A \in \mathfrak{A}$. Notice that $X$ is a convex and by the theorem of Tychonoff compact subset of the locally convex space $E=\mathrm{ba}(\Omega, \mathfrak{U})$ of all bounded additive set functions on $\mathfrak{A}$ under the weak* topology and that the (by [2, V.8.2] nonempty) set $X_{e}$ characterized by the set of the $\{0,1\}$-valued charges on $\mathfrak{A}[1$, p. 245] is weak* closed. The measure $\rho$ has a unique decomposition into a convex combination $\rho=\alpha \rho_{1}+(1-\alpha) \rho_{2}$ of a discrete probability measure $\rho_{1}$ and a probability measure $\rho_{2}$ with $\rho_{2}(\{\nu\})=0$ for all $\nu \in X$, both on the Borel sets of $X$ [2, III.4.14]. Thus $\rho_{1}$ can be represented by $\rho_{1}=\sum_{i=1}^{\infty} \alpha_{i} \varepsilon_{\nu_{i}}$ where $\varepsilon_{\nu_{j}}$ denotes the Dirac measure on the Borel sets of $X$ at $\nu_{j} \in X_{e}$ and where $\alpha_{j} \geqslant 0$ with $\sum_{i=1}^{\infty} \alpha_{i}=1(j \in \mathbf{N})$. Further, the probability charge $\nu^{\prime}$ on $\mathfrak{A}$ defined by $\nu^{\prime}(A)=\int_{X_{e}} \nu(A) \rho_{2}(d \nu)$ for every $A \in \mathfrak{U}$ has the property (*):

Suppose, there is a two-valued charge $\tilde{\nu}$ on $\mathfrak{A}$ with $\nu^{\prime} \geqslant \tilde{\nu}$. Define $\tilde{\nu}=\tilde{\nu} / \tilde{\nu}(\Omega)$, $\Delta=\{A \in \mathfrak{U} \mid \tilde{\tilde{\nu}}(A)=1\}$ and $U_{A}=\left\{\nu \in X_{e} \mid \nu(A)=1\right\}$ for every $A \in \mathfrak{U}$. $U_{A}$ is weak* closed, and for every $A \in \Delta$ the following chain holds:

$$
\begin{aligned}
\nu^{\prime}(A) & =\int_{X_{e}} \nu(A) \rho_{2}(d \nu)=\int_{U_{A}} \nu(A) \rho_{2}(d \nu)+\int_{X_{e} \backslash U_{A}} \nu(A) \rho_{2}(d \nu) \\
& =\rho_{2}\left(U_{A}\right) \geqslant \tilde{\nu}(A)>0
\end{aligned}
$$


and therefore $\inf _{\Delta} \rho_{2}\left(U_{A}\right)>0$. Because $\rho_{2}$ is a bounded regular measure on the Borel sets of a compact space, $\rho_{2}\left(\cap_{\Delta} U_{A}\right)=\inf _{\Delta} \rho_{2}\left(U_{A}\right)$. (Notice that for any $\varepsilon>0$ there is a weak* compact subset $K$ of $\cup_{\Delta} V_{A}$ with $V_{A}=X \backslash U_{A}, A \in \Delta$, such that $\rho_{2}(K)>$ $\rho_{2}\left(\cup_{\Delta} V_{A}\right)-\varepsilon$. Thus, there exists a finite family $\left\{A_{1}, \ldots, A_{n}\right\} \subset \Delta$ with $K \subset \cup_{i=1}^{n} V_{A_{i}}$. Now, define $A_{0}=\bigcap_{i=1}^{n} A_{i}$. Then, $A_{0} \in \Delta$ and $\cup_{i=1}^{n} V_{A_{i}} \subset V_{A_{0}}$ [the first because of $\bigcap_{j=1}^{m} U_{B_{j}}=U_{\cap_{j=1}^{m} B_{j}}$ for all $\left.m \in \mathbf{N}, B_{1}, \ldots, B_{m} \in \mathfrak{A}\right]$. Therefore $\rho_{2}\left(V_{A_{0}}\right) \geqslant \rho_{2}(K)>$ $\rho_{2}\left(\cup_{\Delta} V_{A}\right)-\varepsilon$. Hence $\rho_{2}\left(\cup_{\Delta} U_{A}^{c}\right)=\sup _{\Delta} \rho_{2}\left(U_{A}^{c}\right)$.) Finally, $\cap_{\Delta} U_{A}=\{\tilde{\tilde{\nu}}\}$ holds, which implies $\rho_{2}(\{\tilde{\tilde{\nu}}\})>0$ in contradiction to the property of $\rho_{2}$. Then $\mu(A)=$ $\alpha \sum_{i=1}^{\infty} \alpha_{i} \nu_{i}(A)+(1-\alpha) \nu^{\prime}(A)$ for every $A \in \mathfrak{A}$. Thus the existence of a SobczykHammer decomposition for $\mu$ is established.

Uniqueness. Let $\mu$ be an element of $X$. Then there exists a unique regular probability measure $\rho$ on the Borel sets of $X$ with $\rho\left(X_{e}\right)=1$ which represents $\mu$ (in the sense of $[3$, p. 2]). Suppose $\tilde{\rho}$ is another measure with the same property. Then $\rho\left(U_{A}\right)=\tilde{\rho}\left(U_{A}\right)$ for every $A \in \mathfrak{A}$. Consequently, $\rho$ and $\tilde{\rho}$ coincide on arbitrary unions of sets from $\nabla=\left\{U_{A} \mid A \in \mathfrak{A}\right\}$. (Notice that a similar argument as used in the proof for existence shows $\rho\left(\cap_{\phi} Z_{A}\right)=\inf _{\phi} \rho\left(Z_{A}\right)$ with a subfamily $\phi$ of $\mathfrak{A}$ which is closed with respect to finite unions, and $Z_{A}=\left\{\nu \in X_{e} \mid \nu(A)=0\right\}, A \in \phi$. Paying attention to $\rho\left(X_{e}\right)=\tilde{\rho}\left(X_{e}\right)=1$, this identity implies the preceding coincidence statement.) Because $X_{e}$ is compact with respect to the relative weak* topology and $\nabla$ is a base for this topology $\rho$ and $\tilde{\rho}$ coincide on the Borel sets of $X_{e}$ and therefore also on the Borel sets of $X$.

It follows that the mapping,

$$
\begin{aligned}
& X \rightarrow M_{1}^{+}\left(X_{e}\right), \\
& \mu \rightarrow \rho_{\mu},
\end{aligned}
$$

where $M_{1}^{+}\left(X_{e}\right)$ is the set of all regular probability measures on the Borel sets of $X$ which are concentrated at $X_{e}$ and where $\rho_{\mu}$ denotes the regular probability measure which represents $\mu$, is an affine homeomorphism with (i) $\rho_{\mu}$ is discrete iff $\mu$ has the form $\mu=\sum_{i=1}^{\infty} \mu_{i}$ with at most two-valued charges $\mu_{j}(j \in \mathbf{N})$ and (ii) $\rho_{\mu}$ is continuous, i.e. vanishing at every singleton, iff $\mu$ is continuous. This completes the proof for uniqueness.

REMARKS. (1) To prove the existence of a Sobczyk-Hammer decomposition for a charge $\mu$ on a field $\mathfrak{A}$ of subsets of a set $\Omega$ it also suffices to show that the system $\mathfrak{S}=\left\{\sum_{i=1}^{\infty} \mu_{i} \mid \mu_{j}\right.$ is an at most two-valued charge on $\mathfrak{A}$ for every $j \in \mathbf{N}$ with $\left.\sum_{i=1}^{\infty} \mu_{i} \leqslant \mu\right\}$ is inductively ordered with respect to the usual partial ordering $\leqslant$ on $\mathrm{ba}(\Omega, \mathfrak{A})$, because in that case Zorn's lemma implies the existence of a maximal element $\bar{\mu}$ in $\subseteq$. Thus $\mu$ can be decomposed into $\mu=\bar{\mu}+\mu^{\prime}$ where $\mu^{\prime}=\mu-\bar{\mu} \geqslant 0$. The charge $\mu^{\prime}$ has the property (*).

(2) By the same technique, the following decomposition property can be derived: Let $\mu$ be a charge on a field $\mathfrak{A}$ of subsets of a set $\Omega$. Then $\mu$ can be decomposed into $\mu=\mu_{0}+\mu^{\prime}$ where $\mu_{0}$ is a nonatomic charge on $\mathfrak{A}$ and $\mu^{\prime}$ is a charge on $\mathfrak{A}$ which has no nonnull nonatomic minorant, i.e., $\mu^{\prime}$ has the property $\left(\begin{array}{c}* \\ *\end{array}\right) \mu^{\prime} \geqslant \bar{\mu}_{0}$ implies $\bar{\mu}_{0}=0$ for every nonatomic charge $\bar{\mu}_{0}$ on $\mathfrak{A}$. 
Proof. Consider the system $\subseteq=\{\bar{\mu} \mid \bar{\mu}$ is a nonatomic charge on $\mathfrak{A}$ with $\bar{\mu} \leqslant \mu\}$.

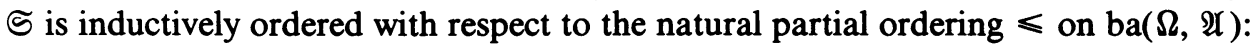
Let $\varsigma_{0}$ be a subset of $\subseteq$ which is linearly ordered. Then the charge $\mu_{0}$ defined by $\mu_{0}(A)=\sup \left\{\bar{\mu}(A) \mid \bar{\mu} \in \mathfrak{S}_{0}\right\}$ for every $A \in \mathfrak{A}$ is nonatomic and therefore an upper bound for $\mathfrak{S}_{0}$ in $\mathfrak{S}$. The nonatomicity of $\mu_{0}$ can be shown by a more canonical indirect argument.

A counterexample shows that in general the preceding decomposition of a charge is not unique:

Define $\Omega=[0,1], \mathbb{S}=\{] a, b] \mid] a, b]$ interval with $] a, b] \subset\left[\frac{1}{4}, \frac{3}{4}[\}\right.$, let $\mathfrak{A}$ denote the field of subsets of $\Omega$ which is generated by $\mathbb{E}$ and let $\mu=\lambda \mid \mathfrak{A}$ be the restriction of the Lebesgue measure $\lambda$ on $\mathfrak{A}$. Then $\mu$ is a nonatomic charge on $\mathfrak{A}$. There are two decompositions for $\mu$ of the above type: First, choose $\mu_{0}=\mu, \mu^{\prime}$ vanishing at every $A \in \mathfrak{A}$. Secondly, choose $\mu^{\prime}$ according to $\mu^{\prime}(B)=\lambda(B \cap A)$ for every $B \in \mathfrak{A}$ where $A=\left[0, \frac{1}{4}\left[\cup\left[\frac{3}{4}, 1\right]\right.\right.$ and $\mu_{0}=\mu-\mu^{\prime}$. Then the following statements hold: (i) $\mu^{\prime}$ is a charge on $\mathfrak{A}$ with $\mu^{\prime} \leqslant \mu$, (ii) $\mu^{\prime}$ is two-valued and therefore has the property ( ${ }_{*}^{*}$ ) because of the fact that $A \subset B$ or $B \subset\left[\frac{1}{4}, \frac{3}{4}\left[\right.\right.$ and thus $\mu^{\prime}(B)=\frac{1}{2}$ or $\mu^{\prime}(B)=0$ for every $B \in \mathfrak{A}$, and (iii) $\mu_{0}$ is nonatomic.

I wish to thank Professor Dr. D. Plachky for valuable remarks.

\section{REFERENCES}

1. G. Choquet, Theory of capacities, Ann. Inst. Fourier (Grenoble) 5 (1955), 131-295.

2. N. Dunford and J. T. Schwartz, Linear operators. Part I: General theory, Interscience, New York, 1958.

3. R. R. Phelps, Lectures on Choquet's theorem, Van Nostrand, Princeton, N. J., 1966.

4. W. Siebe, Vereibbarkeitsuntersuchungen bei Inhalten und Massen, Ph. D. Thesis, University of Münster, Münster, 1979.

5. A. Sobczyk and P. C. Hammer, A decomposition of additive set functions, Duke Math. J. 11 (1944), $839-846$.

6. F. Topsøe, Topology and measure, Springer-Verlag, Berlin and New York, 1970.

INSTITUT FUR MATHEMATISCHE STATISTIK DER UNIVERSITAT MUNSTER, EINSTEINSTR. 62, D-4400 MÚNSTER, Federal RePUblic OF GERMANy 